\title{
Error estimates for a fully discrete $\varepsilon$-uniform finite element method on quasi uniform meshes
}

\author{
Ali Sendur*1 (D), Srinivasan Natesan² (D), Gautam Singh ${ }^{3}$ (D) \\ ${ }^{1}$ Department of Mathematics, Alanya Alaaddin Keykubat University, Antalya, 07430, Turkey \\ ${ }^{2,3}$ Department of Mathematics, Indian Institute of Technology, Guwahati 781039, India
}

\begin{abstract}
In this article, we analyze a fully discrete $\varepsilon$-uniformly convergent finite element method for singularly perturbed convection-diffusion-reaction boundary-value problems, on piecewiseuniform meshes. Here, we choose $L$-splines as basis functions. We will concentrate on the convergence analysis of the finite element method which employ the discrete $L$-spline basis functions instead of their continuous counterparts. The $L$-splines are approximated on the piecewise-uniform Shishkin mesh inside each element. These approximations are used as basis functions in the frame of Galerkin FEM on a coarse piecewise-uniform mesh to discretize the domain. Further, we determine the amount of error introduced by the discrete $L$-spline basis functions in the overall numerical method, and explore the possibility of recovering the order of convergence that are consistent with the classical order of convergence for the numerical methods using the exact $L$-splines.
\end{abstract}

Mathematics Subject Classification (2020). 65L11, 65L60, 65L70, 65N30

Keywords. singularly perturbed convection-diffusion-reaction boundary-value problem, finite element method, boundary layers, piecewise-uniform Shishkin mesh, uniform convergence

\section{Introduction}

In this article, we analyze a fully discrete $\varepsilon$-uniformly convergent finite element method (FEM) for the following singularly perturbed two-point boundary-value problem, on piecewiseuniform meshes:

$$
\left\{\begin{array}{l}
L u=-\varepsilon u^{\prime \prime}+b(x) u^{\prime}+c(x) u=f(x), \quad x \in \Omega=(0,1), \\
u(0)=u(1)=0,
\end{array}\right.
$$

where $0<\varepsilon \ll 1$ is the diffusion coefficient, $b(x) \in C^{2}(\bar{\Omega}), c(x) \in C^{1}(\bar{\Omega}), f(x) \in C^{1}(\bar{\Omega})$, and for $x \in \bar{\Omega}$, we have

$$
b(x) \geq \alpha>0 .
$$

\footnotetext{
* Corresponding Author.

Email addresses: ali.sendur@alanya.edu.tr (A. Sendur), natesan@iitg.ac.in (S. Natesan), gautamsingh@iitg.ac.in (G. Singh)

Received: 19.02.2020; Accepted: 06.04.2021
} 
We note that, by changing the variable in problem (1.1), in particular employing $v=e^{-\sigma x} u$ with suitably chosen $\sigma$, we may assume that there exists a constant $C>0$ such that for $x \in \bar{\Omega}$ we have

$$
c(x)-\frac{1}{2} b^{\prime}(x) \geq C,
$$

which means that, there is no essential loss in generality by assuming (1.3) rather than the condition (1.2). See [28] for more details.

It is well-known that the classical finite element methods (FEMs) using conforming piecewise polynomial basis functions for the numerical solution of singularly perturbed boundary-value problems (BVP) of the form (1.1) are not effective in the critical range when the parameter $0<\varepsilon \ll 1$ is considerably small compared to the mesh parameter $h$ [27]. That deficiency disappears if we add a sufficient amount of artificial diffusion to the problem through an appropriate stabilized FEM and the resulting numerical method produces an approximation consistent with the physical configuration of the problem $[1$, 5,9]. For other attempts to derive improved discretizations, we refer to the Residual-Free Bubbles (RFB) method [4,10,14,15], the Variational Multiscale (VMS) method $[11,17,18]$ and the Discontinuous Galerkin finite element method (DGFEM) $[6,8]$. However, the numerical approximation may still not converge uniformly to the exact solution of the orginal problem in the layer region(s). Hence, it is important to construct $\varepsilon$-uniformly convergent methods ensuring the consistency of the numerical solutions with the exact solution of the problem in the whole range throughout the entire domain.

There are two major approaches for the construction of $\varepsilon$-uniformly convergent FEMs: The first of these involves the use of special piecewise-uniform meshes, such as Shishkin mesh which is adapted according to the singular perturbation nature of the problem [20, $22,26,29]$. The Shishkin mesh consists of coupling two uniform meshes having different mesh parameters on each side of a transition point. It turns out that the Shishkin mesh applied in the frame of the Galerkin FEM is sufficient for the construction of $\varepsilon$-uniform method (see [24]). The use of equidistant subintervals on each side of the transition point and its simplicity can be considered as a major attraction of this approach. However, it requires prior information about the location and width of the layer.

Yet another approach for the construction of $\varepsilon$-uniform FEM is based on enriching the finite element space whose basis functions reflect the nature of the problem through a local differential equation. We will concentrate on the FEM whose basis functions are the so-called $L$-splines on piecewise-uniform meshes. This approach is closely related with the RFB method whose function space is enriched by the residual-free bubble functions. For more details about the applications, implementation and convergence properties of the RFB method one can refer $[2-4,13,25]$. FEMs using $L$-splines have the desired property that the method uses a coarse mesh and requires no a priori information about the width and location of the boundary layer. However, the drawback of this methodology resides in that it requires to solve the locally defined differential equation which has similar characteristic behavior of the original one. Therefore, typical implementation of such methods is based on approximating the $L$-splines by an appropriate numerical method inside each element and using these approximations (let us call them the discrete $L$-spline functions) in place of the $L$-splines $[7,12,13]$. It is usual to expect that the use of the appropriate approximations to $L$-splines in the numerical method produces small errors that are negligible. However, it is not clear how much error the use of discrete $L$-splines contributes to the overall numerical method. On the other hand, that may be an important point to balance and optimize the number of nodes at the global level and the mesh used to approximate $L$-splines inside each element.

This work aims to reveal the convergence properties of the numerical method which uses discrete $L$-spline basis functions in place of $L$-splines on a piecewise-uniform mesh. To this end, we first consider a coarse, piecewise-uniform mesh and then partition each element 
into a mesh of Shishkin type depending on the local flow regime. We use the Galerkin FEM with piecewise linears to approximate $L$-spline basis functions on the local mesh. Then we derive the error bounds between $L$-splines and their approximate counterparts. Finally, we employ the discrete $L$-splines as basis functions to solve the problem on the coarse mesh in the context of Galerkin FEM and prove that the resulting numerical method converges uniformly in the $\varepsilon$-weighted energy norm. Here, we confine our study to one-dimensional problem only, because it involves most of the major difficulties that can be confronted in higher dimensions, and also it is technically good enough.

The layout of the paper is as follows: We provide the bounds for the solution of the continuous problem (1.1) in Section 2. We introduce the proposed numerical method and all its ingredients, like the mesh, finite element space, basis functions, etc. in Section 3. Element-wise error bounds between $L$-splines and discrete $L$-splines are presented in Section 4.1. However, their proofs are shifted to and given in detail in Appendix for the sake of convenience. In Section 4.2, we recall some classical results from the interpolation theory and further derive some interpolation error estimates when the interpolation space is spanned by the discrete $L$-spline functions. Finally, we obtain the main result in Section 4.3 in which we prove that the approximate solution obtained by the proposed numerical method converges uniformly with respect to $\varepsilon$.

Throughout this article, $C$ denotes a generic positive constant which is independent of the parameter $\varepsilon$ and the number of mesh points.

\section{Bounds for the solution of singularly perturbed problem (1.1)}

The existence and uniqueness of the solution of the BVP (1.1) is well-known [16]. We recall some properties of (1.1) which we will need later for the analysis of the numerical method.

Lemma 2.1. The solution $u$ of the BVP (1.1) satisfies the following bounds:

$$
\|u\|_{\infty} \leq C, \quad\left|u^{(k)}(x)\right| \leq C\left(1+\varepsilon^{-k} e^{-\alpha(1-x) / \varepsilon}\right)
$$

for $0<x<1$ and $0 \leq k \leq 3$.

Proof. The proof of this lemma can be found in [19].

\section{The numerical method}

In this section, we introduce the numerical method along with all of its ingredients, like, mesh points, finite element space, basis functions, etc.

To begin with, we define a regular mesh $\bar{\Omega}^{N}=\left\{x_{i}\right\}_{i=0}^{N}$, where $0=x_{0}<x_{1}<\ldots<x_{N}=1$ and set $h_{i}=x_{i}-x_{i-1}$ for $i=1, \ldots, N$, with $h=\max _{1 \leq i \leq N} h_{i}$. For $i=1, \ldots, N-1$, set $\bar{h}_{i}=\left(h_{i}+h_{i+1}\right) / 2$. To proceed, we assume the quasi-uniformity of the grid, i.e., there exists a constant $C_{0}>0$ such that

$$
C_{0} h \leq h_{i} \leq h=\max _{1 \leq i \leq N} h_{i} .
$$

We approximate $b(x)$ by piecewise constant function $\bar{b}(x)$ which is defined by,

$$
\bar{b}(x)=\bar{b}_{i}=\frac{b\left(x_{i-1}\right)+b\left(x_{i}\right)}{2} \text { in } \Omega_{i}=\left(x_{i-1}, x_{i}\right) .
$$

In a similar way, we define $\bar{c}$ and $\bar{f}$.

Definitions and notations. We fix notation that will be used throughout the paper. Let $(\cdot, \cdot)$ denote the usual $L_{2}(\Omega)$ inner product and define $(\cdot, \cdot)_{d}$ to be its discrete counterpart, that is,

$$
(v, w)_{d}=\sum_{i=1}^{N-1} \bar{h}_{i} v\left(x_{i}\right) w\left(x_{i}\right) .
$$


Similarly, let $\|\cdot\|$ be the usual $L_{2}(\Omega)$-norm and define the discrete $L_{2}(\Omega)-$ norm $\|\cdot\|_{d}$ by

$$
\|w\|_{d}=\left\{\sum_{i=1}^{N-1} \bar{h}_{i} w^{2}\left(x_{i}\right)\right\}^{1 / 2} .
$$

Then the corresponding energy norms are defined to be $\|p\|_{\varepsilon}^{2}=\varepsilon\left\|p^{\prime}\right\|^{2}+\|p\|^{2}$ and $\|p\|_{\varepsilon, d}^{2}=\varepsilon\left\|p^{\prime}\right\|^{2}+\|p\|_{d}^{2}$. We denote the $\infty-$ norm of $p$ over $\Omega$ by $\|\cdot\|_{\infty}$ and we also use the notations $\|\cdot\|_{K},(\cdot, \cdot)_{K},\|\cdot\|_{\infty, K},\|\cdot\|_{\varepsilon, K},\|\cdot\|_{\varepsilon, d, K}$ when the integrals in the definitions of $\|\cdot\|,(\cdot, \cdot),\|\cdot\|_{\infty},\|\cdot\|_{\varepsilon},\|\cdot\|_{\varepsilon, d}$ are restricted to the interval $K$. Set

$$
\bar{a}(p, q)=\varepsilon\left(p^{\prime}, q^{\prime}\right)+\left(\bar{b} p^{\prime}, q\right)+\sum_{i=1}^{N-1} \bar{h}_{i}(c p q)\left(x_{i}\right) .
$$

A lumping process is used in (3.1) to simplify calculations without causing any loss of generality. We shall further say that a quantity $q$ is $O(z)$ when $|q| \leq C z$ holds for any sufficiently small $z$. We finally note that, we will use the following arithmetic-geometric mean inequality (Young's inequality) repeatedly:

$$
y z \leq \gamma y^{2}+\frac{z^{2}}{4 \gamma}, \quad \gamma>0 .
$$

\subsection{Standard FEM with $L$-spline basis functions}

Now we can define $L$-spline basis functions $\varphi_{i}$, which span the finite element space $V_{h} \subset H_{0}^{1}(\Omega)$, for each $i=1, \ldots, N-1$, by

$$
\left\{\begin{array}{l}
-\varepsilon \varphi_{i}^{\prime \prime}+\bar{b} \varphi_{i}^{\prime}+\bar{c} \varphi_{i}=0, \quad \text { on } \Omega_{i} \cup \Omega_{i+1} \\
\varphi_{i}\left(x_{j}\right)=\delta_{i j},
\end{array}\right.
$$

where $\bar{b} \geq \alpha_{i}>0, \quad \bar{c} \geq \beta_{i} \quad$ in $\Omega_{\mathrm{i}}$ and

$$
H_{0}^{1}(\Omega)=\left\{u \in L_{2}(\Omega): u^{\prime} \in L_{2}(\Omega) \text { with } u(0)=u(1)=0\right\} .
$$

The standard Galerkin FEM for the BVP (1.1) is based upon the variational formulation. It consists of taking the finite dimensional subspace $V_{h}$ of $H_{0}^{1}(\Omega)$ associated with the partition $\bar{\Omega}^{N}$ of the domain $\Omega$ and then solving the corresponding problem:

$$
\left\{\begin{array}{l}
\text { Find } u_{h} \in V_{h}, \text { such that } \\
\bar{a}\left(u_{h}, v_{h}\right)=\left(f, v_{h}\right)_{d}, \quad \forall v_{h} \in V_{h} .
\end{array}\right.
$$

The error of the standard Galerkin FEM using $L$-spline functions satisfies the following estimate:

Theorem 3.1. Let $u$ and $u_{h}$ be respectively the solutions of the continuous and discrete problems (1.1) and (3.4), where $V_{h}$ is the space of fully exponentially-fitted L-splines. Then the error satisfies the following uniform estimate:

$$
\left\|u-u_{h}\right\|_{\varepsilon, d} \leq C h^{1 / 2} .
$$

Proof. See [28] for details.

The convergence analysis of such numerical algorithms is also based on the exact solutions of $L$-spline basis functions [24]. However, in practical computations, either it is difficult to find the exact solution of (3.3) or it is not practical to solve it from the implementation point of view [7]. It is indeed reasonable to expect a qualitatively similar numerical results by employing convenient approximations $\varphi_{i}^{*}$ to $L$-splines and using them as basis functions in lieu of $\varphi_{i}$. However, it is not clear the cost of using the discrete counterparts of $L$-splines as basis functions to the overall numerical algorithm, which we will now investigate. 


\subsection{Standard FEM with discrete $L$-spline basis functions}

For the counterpart of (3.4) which uses the discrete $L$-spline basis functions, we start by explaining how we get approximate solution $\varphi_{i}^{*}$ of $\varphi_{i}$ in an appropriate sense. Take the support of $\varphi_{i}$, denote it by $\operatorname{supp} \varphi_{i}=\bar{\Omega}_{i} \cup \bar{\Omega}_{i+1}$ and divide it into the four subintervals $\left[x_{i-1}, x_{i}-\tau_{i}\right],\left[x_{i}-\tau_{i}, x_{i}\right],\left[x_{i}, x_{i+1}-\tau_{i+1}\right],\left[x_{i+1}-\tau_{i+1}, x_{i+1}\right]$, each has $M / 2$ mesh elements, where

$$
\tau_{i}=\min \left\{\frac{h_{i}}{2}, \frac{2 \varepsilon}{\alpha_{i}} \ln \left(\frac{M}{h_{i}}\right)\right\} .
$$

The Shishkin's mesh $\Omega_{i, \tau_{i}}^{M}$ is defined inside each element $\bar{\Omega}_{i}$ by

$$
x_{i, 0}^{*}=x_{i-1} \quad \text { and } \quad h_{k}^{*}=x_{i, k}^{*}-x_{i, k-1}^{*}= \begin{cases}\frac{2\left(h_{i}-\tau_{i}\right)}{M}, & 0<k \leq M / 2 \\ \frac{2 \tau_{i}}{M}, & M / 2<k \leq M .\end{cases}
$$

We shall assume that $\tau_{i}=\frac{2 \varepsilon}{\alpha_{i}} \ln \left(M / h_{i}\right)$, otherwise $\varepsilon \geq \frac{\alpha_{i} h_{i}}{4 \ln \left(M / h_{i}\right)}$ in which case the parameter $\varepsilon$ is not so small and therefore the problem can be analyzed in the classical way, which is not of interest here.

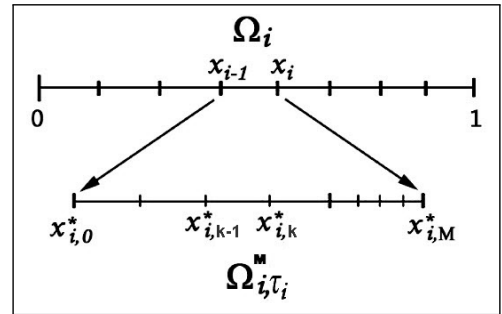

Figure 1. Shishkin mesh on $\Omega_{i}$

The discrete $L$-spline basis functions $\varphi_{i}^{*}$ are used on the pair of $\bar{\Omega}_{i}$ and $\bar{\Omega}_{i+1}$ within the context of the standard Galerkin FEM. Here, the finite element spaces $W_{i}^{*}\left(\Omega_{i, \tau_{i}}^{M}\right)$ and $W_{i+1}^{*}\left(\Omega_{i+1, \tau_{i+1}}^{M}\right)$ are spanned by the continuous piecewise linear basis functions (see Appendix for details). Now we can define $V_{h}^{*}$ to be the finite element space spanned by the discrete $L$-spline basis functions $\varphi_{i}^{*}$. Thus the counterpart of the discrete problem (3.4) on $\bar{\Omega}^{N}$ reads:

$$
\left\{\begin{array}{l}
\text { Find } u_{h}^{*} \in V_{h}^{*}, \text { such that } \\
\bar{a}\left(u_{h}^{*}, v_{h}^{*}\right)=\left(f, v_{h}^{*}\right)_{d}, \quad \forall v_{h}^{*} \in V_{h}^{*} .
\end{array}\right.
$$

Now, we shall prove that $\bar{a}(\cdot, \cdot)$ is $\varepsilon$-uniformly coercive on the space $V_{h}^{*}$ relative to $\|\cdot\|_{\varepsilon, d}$.

Lemma 3.2. Let $\bar{a}(\cdot, \cdot)$ be the bilinear form in (3.1). Then, there exists an $h_{0}$, independent of $\varepsilon$, such that for all $h, 0<h \leq h_{0}$ and for any function $v_{h}^{*} \in V_{h}^{*}$

$$
\bar{a}\left(v_{h}^{*}, v_{h}^{*}\right) \geq \beta\left\|v_{h}^{*}\right\|_{\varepsilon, d}^{2} .
$$

where $\beta$ is a positive constant that is independent of the parameter $\varepsilon$ and of the number of mesh intervals $N$.

Proof. Using the definition of the discrete bilinear form, $\bar{a}(\cdot, \cdot)$, we have

$$
\bar{a}\left(v_{h}^{*}, v_{h}^{*}\right)=\varepsilon\left(v_{h}^{* \prime}, v_{h}^{* \prime}\right)+\left(\bar{b} v_{h}^{* \prime}, v_{h}^{*}\right)+\sum_{i=1}^{N-1} \bar{h}_{i} c\left(x_{i}\right) v_{h}^{* 2}\left(x_{i}\right)
$$


for any $v_{h}^{*} \in V_{h}^{*}$. But the second term on the right hand side can be expressed in the following form

$$
\begin{aligned}
\left(\bar{b} v_{h}^{* \prime}, v_{h}^{*}\right) & =\sum_{i=1}^{N} \bar{b} \sum_{k=1}^{M} \int_{x_{i, k-1}^{*}}^{x_{i, k}^{*}} \frac{1}{2}\left(v_{h}^{* 2}\right)^{\prime} d x=\frac{1}{2} \sum_{i=1}^{N} \bar{b}\left(v_{h}^{* 2}\left(x_{i}\right)-v_{h}^{* 2}\left(x_{i-1}\right)\right) \\
& =-\frac{1}{2} \sum_{i=1}^{N-1} \frac{b\left(x_{i+1}\right)-b\left(x_{i-1}\right)}{2} v_{h}^{* 2}\left(x_{i}\right) \\
& =-\frac{1}{2} \sum_{i=1}^{N-1}\left(\bar{h}_{i} b^{\prime}\left(x_{i}\right)+O\left(h_{i}^{2}+h_{i+1}^{2}\right)\right) v_{h}^{* 2}\left(x_{i}\right) .
\end{aligned}
$$

Thus, we have

$$
\bar{a}\left(v_{h}^{*}, v_{h}^{*}\right)=\varepsilon\left(v_{h}^{* \prime}, v_{h}^{* \prime}\right)+\sum_{i=1}^{N-1}\left(\bar{h}_{i}\left(c\left(x_{i}\right)-\frac{1}{2} b^{\prime}\left(x_{i}\right)\right)+O\left(h_{i}^{2}+h_{i+1}^{2}\right)\right) v_{h}^{* 2}\left(x_{i}\right) .
$$

But condition (1.3) implies that for some $h_{0}$, independent of $\varepsilon$, and for all $0<h \leq h_{0}$, we have

$$
\bar{h}_{i}\left(c\left(x_{i}\right)-\frac{1}{2} b^{\prime}\left(x_{i}\right)\right)+O\left(h_{i}^{2}+h_{i+1}^{2}\right) \geq C_{1} \bar{h}_{i} .
$$

Therefore, for all $h \in\left(0, h_{0}\right]$, we have

$$
\bar{a}\left(v_{h}^{*}, v_{h}^{*}\right) \geq \beta\left\|v_{h}^{*}\right\|_{\varepsilon, d}^{2} .
$$

This completes the proof.

\section{Convergence analysis}

In this section, we study the convergence features of the proposed method (3.7) and show that a priori error estimates of the method (3.4) continue to hold for the proposed algorithm. To do so, we first present some preliminary results on error estimates between $L$-spline basis function $\varphi_{i}$ and its discrete counterpart $\varphi_{i}^{*}$ in a typical element $\bar{\Omega}_{i}$. We will use these estimates to obtain the interpolation error bounds in Section 4.2 which will be our basic tools to prove the main convergence theorem in Section 4.3.

\subsection{Error bounds on basis functions}

The local convergence estimates will be obtained in two different norms. Their proofs are given in Appendix A for the sake of convenience. Denoting the restriction $\varphi_{i}$ to the element $\bar{\Omega}_{i}$ by $\varphi_{i, \Omega_{i}}$, we have the following:

Lemma 4.1. Let $\varphi_{i, \Omega_{i}}$ be solution of problem (3.3), and $\varphi_{i, \Omega_{i}}^{*}$ be solution of problem (A.14). Then, we have

$$
\left\|\varphi_{i, \Omega_{i}}^{*}-\varphi_{i, \Omega_{i}}\right\|_{\infty, \Omega_{i}} \leq h_{i} \gamma_{i, \infty}^{*}(M), \quad \text { where } \quad \gamma_{i, \infty}^{*}(M)=C M^{-\frac{1}{2}}\left(\ln \left(M / h_{i}\right)\right)^{2} .
$$

The following lemma gives an $\varepsilon$-uniform estimate in the energy norm:

Lemma 4.2. Let $\varphi_{i, \Omega_{i}}$ be solution of problem (3.3) and $\varphi_{i, \Omega_{i}}^{*}$ be solution of problem (A.14). Then, we have

$$
\left\|\varphi_{i, \Omega_{i}}^{*}-\varphi_{i, \Omega_{i}}\right\|_{\varepsilon, \Omega_{i}} \leq h_{i} \gamma_{i, \varepsilon}^{*}(M) \quad \text { where } \quad \gamma_{i, \varepsilon}^{*}(M)=C M^{-1}\left(\ln \left(M / h_{i}\right)\right)^{2} .
$$




\subsection{Error estimates on interpolation}

Here we recall some well-known results from the interpolation theory and derive new error estimates for the interpolation functions spanned by the discrete $L$-spline basis functions.

Lemma 4.3. Let $u_{I}$ denote the interpolation of $u$ in the space $V_{h}$, that $i s, u_{I}\left(x_{i}\right)=u\left(x_{i}\right)$, $\forall x_{i} \in \bar{\Omega}^{N}$, and $u_{I} \in V_{h}$. Then we have

$$
\left\|u-u_{I}\right\|_{\infty} \leq C h, \quad\left\|u-u_{I}\right\|_{\varepsilon, d} \leq C h^{1 / 2} .
$$

Proof. See $[23,24]$ for the detailed proof.

An $\varepsilon$-uniform error estimate for the interpolation function $u_{I}^{*}$ spanned by discrete $L$-spline basis functions in maximum norm is given in the next lemma.

Lemma 4.4. Let $u_{I}^{*}$ denote the interpolation of $u$ in the space $V_{h}^{*}$; that $i s, u_{I}^{*}\left(x_{i}\right)=u\left(x_{i}\right)$, $\forall x_{i} \in \bar{\Omega}^{N}$, and $u_{I}^{*} \in V_{h}^{*}$. Then we have

$$
\left\|u-u_{I}^{*}\right\|_{\infty} \leq C h\left(1+\gamma_{\infty}^{*}(M)\right) \text {, where } \gamma_{\infty}^{*}(M)=M^{-\frac{1}{2}}(\ln (M / h))^{2} .
$$

Proof. In the light of Lemma 4.3, we have

$$
\left\|u-u_{I}^{*}\right\|_{\infty} \leq\left\|u-u_{I}\right\|_{\infty}+\left\|u_{I}-u_{I}^{*}\right\|_{\infty} \leq C h+\left\|u_{I}-u_{I}^{*}\right\|_{\infty} .
$$

On the other hand, for any $x \in \Omega_{i}$, we have

$$
\begin{aligned}
\left(u_{I}(x)-u_{I}^{*}(x)\right)= & \left(u\left(x_{i-1}\right) \varphi_{i-1, \Omega_{i}}(x)+u\left(x_{i}\right) \varphi_{i, \Omega_{i}}(x)\right) \\
& \quad-\left(u\left(x_{i-1}\right) \varphi_{i-1, \Omega_{i}}^{*}(x)+u\left(x_{i}\right) \varphi_{i, \Omega_{i}}^{*}(x)\right) \\
\leq & \left|u\left(x_{i-1}\right)\left(\varphi_{i-1, \Omega_{i}}(x)-\varphi_{i-1, \Omega_{i}}^{*}(x)\right)+u\left(x_{i}\right)\left(\varphi_{i, \Omega_{i}}(x)-\varphi_{i, \Omega_{i}}^{*}(x)\right)\right| \\
\leq & \max \left\{\left|u\left(x_{i-1}\right)\right|,\left|u\left(x_{i}\right)\right|\right\}\left(\left\|\varphi_{i-1, \Omega_{i}}^{*}-\varphi_{i-1, \Omega_{i}}\right\|_{\infty, \Omega_{i}}\right. \\
& \left.\quad+|| \varphi_{i, \Omega_{i}}^{*}-\varphi_{i, \Omega_{i}} \|_{\infty, \Omega_{i}}\right) \\
\leq & \max \left\{\left|u\left(x_{i-1}\right)\right|,\left|u\left(x_{i}\right)\right|\right\}\left(h_{i-1} \gamma_{i-1, \infty}^{*}(M)+h_{i} \gamma_{i, \infty}^{*}(M)\right),
\end{aligned}
$$

where we have used Lemma 4.1. Finally, we get

$$
\left\|u_{I}-u_{I}^{*}\right\|_{\infty}=\sup _{i}\left\|u_{I}-u_{I}^{*}\right\|_{\infty, \Omega_{i}} \leq C h_{i} \sup _{i}\|u\|_{\infty, \Omega_{i}} \gamma_{i, \infty}^{*}(M) \leq C h\|u\|_{\infty} \gamma_{\infty}^{*}(M),
$$

where $\gamma_{\infty}^{*}(M)=\max _{1 \leq i \leq N-1} \gamma_{i, \infty}^{*}(M)$. We get the desired estimate by combining (4.1) with the bound for $\left\|u_{I}-u_{I}^{*}\right\|_{\infty}$.

The next lemma gives an $\varepsilon$-uniform error estimate in the discrete energy norm:

Lemma 4.5. Let $u_{I}^{*}$ be the nodal interpolant of $u$ in $V_{h}^{*}$. Then we have

$$
\left\|u-u_{I}^{*}\right\|_{\varepsilon, d} \leq C h^{1 / 2}\left(1+\gamma_{\varepsilon}^{*}(M)\right) \text {, where } \gamma_{\varepsilon}^{*}(M)=M^{-1}(\ln (M / h))^{2} .
$$

Proof. Using the triangle inequality and Lemma 4.3, we have

$$
\left\|u-\left.u_{I}^{*}\right|_{\varepsilon, d} \leq\right\| u-u_{I}\left\|_{\varepsilon, d}+\right\| u_{I}-u_{I}^{*}\left\|_{\varepsilon, d} \leq C h^{1 / 2}+\right\| u_{I}-u_{I}^{*} \|_{\varepsilon, d} .
$$

To get a bound on $\left\|u_{I}-u_{I}^{*}\right\|_{\varepsilon, d}$, observe that

$$
\left\|u_{I}-u_{I}^{*}\right\|_{\varepsilon, d}^{2}=\varepsilon\left(\left(u_{I}-u_{I}^{*}\right)^{\prime},\left(u_{I}-u_{I}^{*}\right)^{\prime}\right)+\left(u_{I}-u_{I}^{*}, u_{I}-u_{I}^{*}\right)_{d}=\mathcal{J}+\mathcal{J J} .
$$

The estimate of JJ is straightforward:

$$
\mathcal{J J}=\left(u_{I}-u_{I}^{*}, u_{I}-u_{I}^{*}\right)_{d}=\sum_{i=1}^{N-1} \bar{h}_{i}\left(u_{I}-u_{I}^{*}\right)^{2}\left(x_{i}\right)=0 .
$$


For the diffusive term, we have

$$
\begin{aligned}
\mathcal{J} & =\varepsilon\left(\left(u_{I}-u_{I}^{*}\right)^{\prime},\left(u_{I}-u_{I}^{*}\right)^{\prime}\right)=\varepsilon \sum_{i=1}^{N} \int_{x_{i-1}}^{x_{i}}\left(u_{I}-u_{I}^{*}\right)^{\prime 2} d x \\
& =\varepsilon \sum_{i=1}^{N} \int_{x_{i-1}}^{x_{i}}\left[u\left(x_{i-1}\right)\left(\varphi_{i-1, \Omega_{i}}-\varphi_{i-1, \Omega_{i}}^{*}\right)^{\prime}+u\left(x_{i}\right)\left(\varphi_{i, \Omega_{i}}-\varphi_{i, \Omega_{i}}^{*}\right)^{\prime}\right]^{2} d x \\
& \leq 2 \varepsilon\|u\|_{\infty}^{2} \sum_{i=1}^{N} \int_{x_{i-1}}^{x_{i}}\left(\left[\left(\varphi_{i-1, \Omega_{i}}-\varphi_{i-1, \Omega_{i}}^{*}\right)^{\prime}\right]^{2}+\left[\left(\varphi_{i, \Omega_{i}}-\varphi_{i, \Omega_{i}}^{*}\right)^{\prime}\right]^{2}\right) d x \\
& \leq 2\|u\|_{\infty}^{2} \sum_{i=1}^{N}\left(\left\|\varphi_{i-1, \Omega_{i}}-\varphi_{i-1, \Omega_{i}}^{*}\right\|_{\varepsilon, \Omega_{i}}^{2}+\left\|\varphi_{i, \Omega_{i}}-\varphi_{i, \Omega_{i}}^{*}\right\|_{\varepsilon, \Omega_{i}}^{2}\right) \\
& \leq 2\|u\|_{\infty}^{2} \sum_{i=1}^{N}\left(h_{i-1}^{2} \gamma_{i-1, \varepsilon}^{* 2}(M)+h_{i}^{2} \gamma_{i, \varepsilon}^{* 2}(M)\right) \\
& \leq C h\|u\|_{\infty}^{2} \gamma_{\varepsilon}^{* 2}(M),
\end{aligned}
$$

where we have used the Cauchy-Schwarz inequality, Lemma 4.2 and $\gamma_{\varepsilon}^{*}(M)=\max _{1 \leq i \leq N-1} \gamma_{i, \varepsilon}^{*}(M)$. Substituting $\mathcal{J}$ and $\mathcal{J} \mathcal{J}$ in (4.3), we obtain that

$$
\left\|u_{I}-u_{I}^{*}\right\|_{\varepsilon, d} \leq C h^{1 / 2}\|u\|_{\infty} \gamma_{\varepsilon}^{*}(M)
$$

Combining this estimate with (4.2) and Lemma 2.1, gives the desired result.

\subsection{Convergence of the numerical method}

In this section, we will prove that the approximate solution obtained through the proposed numerical method converges uniformly in $\varepsilon$. We first deduce some inequalities which will be required for the proof of the main theorem.

Lemma 4.6. Let $u$ be the solution of (1.1) and $u_{h}^{*}$ be the solution of (3.7). Then, we have

$$
\bar{a}\left(u-u_{h}^{*}, v_{h}^{*}\right) \leq C h\left\|v_{h}^{*}\right\|_{d}
$$

for any $v_{h}^{*} \in V_{h}^{*}$.

Proof. Since $v_{h}^{*} \in V_{h}^{*}$, it can be expressed in the following form:

$$
v_{h}^{*}=\sum_{i=1}^{N-1} v_{i}^{*} \varphi_{i, \Omega_{i}}^{*}
$$

where $v_{i}^{*}=v_{h}^{*}\left(x_{i}\right)$. We have

$$
\bar{a}\left(u-u_{h}^{*}, v_{h}^{*}\right)=\sum_{i=1}^{N-1} v_{i}^{*} \bar{a}\left(u-u_{h}^{*}, \varphi_{i, \Omega_{i}}^{*}\right) .
$$


Now observe that

$$
\begin{aligned}
\varepsilon\left(u^{\prime}, \varphi_{i, \Omega_{i}}^{* \prime}\right) & =\varepsilon \sum_{j=1}^{N} \int_{x_{j-1}}^{x_{j}} u^{\prime} \varphi_{i, \Omega_{i}}^{* \prime} d x=\varepsilon \sum_{j=1}^{N} \sum_{k=1}^{M} \int_{x_{j, k-1}^{*}}^{x_{j, k}^{*}} u^{\prime} \varphi_{i, \Omega_{i}}^{* \prime} d x \\
& =\varepsilon \sum_{j=1}^{N} \sum_{k=1}^{M}\left(\left.u^{\prime} \varphi_{i, \Omega_{i}}^{*}\right|_{x_{j, k-1}^{*}} ^{x_{j, k}^{*}}-\int_{x_{j, k-1}^{*}}^{x_{j, k}^{*}} u^{\prime \prime} \varphi_{i, \Omega_{i}}^{*} d x\right) \\
& =\varepsilon \sum_{j=1}^{N}\left(u^{\prime}\left(x_{j}\right) \varphi_{i, \Omega_{i}}^{*}\left(x_{j}\right)-u^{\prime}\left(x_{j-1}\right) \varphi_{i, \Omega_{i}}^{*}\left(x_{j-1}\right)-\int_{x_{j-1}}^{x_{j}} u^{\prime \prime} \varphi_{i, \Omega_{i}}^{*} d x\right) \\
& =\varepsilon\left(u^{\prime}\left(x_{N}\right) \varphi_{i, \Omega_{i}}^{*}\left(x_{N}\right)-u^{\prime}\left(x_{0}\right) \varphi_{i, \Omega_{i}}^{*}\left(x_{0}\right)-\int_{0}^{1} u^{\prime \prime} \varphi_{i, \Omega_{i}}^{*} d x\right) \\
& =-\varepsilon \int_{0}^{1} u^{\prime \prime} \varphi_{i, \Omega_{i}}^{*} d x=-\varepsilon\left(u^{\prime \prime}, \varphi_{i, \Omega_{i}}^{*}\right),
\end{aligned}
$$

and that

$$
\begin{aligned}
\left(c u-f, \varphi_{i, \Omega_{i}}^{*}\right)_{d} & =\sum_{j=1}^{N-1} \bar{h}_{j}\left(c\left(x_{j}\right) u\left(x_{j}\right)-f\left(x_{j}\right)\right) \varphi_{i, \Omega_{i}}^{*}\left(x_{j}\right)=\bar{h}_{i}\left(c\left(x_{i}\right) u\left(x_{i}\right)-f\left(x_{i}\right)\right) \\
& =\left(c\left(x_{i}\right) u\left(x_{i}\right)-f\left(x_{i}\right), \varphi_{i, \Omega_{i}}^{*}\right)
\end{aligned}
$$

where we have used the fact that $\left(1, \varphi_{i, \Omega_{i}}^{*}\right)=\bar{h}_{i}$. We note that, for each $i, 1 \leq i \leq N-1$, we have

$$
\begin{aligned}
\bar{a}\left(u-u_{h}^{*}, \varphi_{i, \Omega_{i}}^{*}\right) & =\bar{a}\left(u, \varphi_{i, \Omega_{i}}^{*}\right)-\bar{a}\left(u_{h}^{*}, \varphi_{i, \Omega_{i}}^{*}\right)=\bar{a}\left(u, \varphi_{i, \Omega_{i}}^{*}\right)-\left(f, \varphi_{i, \Omega_{i}}^{*}\right)_{d} \quad(\text { by }(3.7)) \\
& =\varepsilon\left(u^{\prime}, \varphi^{* \prime}{ }_{i, \Omega_{i}}\right)+\left(\bar{b} u^{\prime}, \varphi_{i, \Omega_{i}}^{*}\right)+\left(c u, \varphi_{i, \Omega_{i}}^{*}\right)_{d}-\left(f, \varphi_{i, \Omega_{i}}^{*}\right)_{d} \\
& =\left(-\varepsilon u^{\prime \prime}, \varphi_{i, \Omega_{i}}^{*}\right)+\left(\bar{b} u^{\prime}, \varphi_{i, \Omega_{i}}^{*}\right)+\left(c u-f, \varphi_{i, \Omega_{i}}^{*}\right)_{d} \quad(\operatorname{by}(4.7)) \\
(\operatorname{by}(1.1),(4.8)) & =\left(f-b u^{\prime}-c u, \varphi_{i, \Omega_{i}}^{*}\right)+\left(\bar{b} u^{\prime}, \varphi_{i, \Omega_{i}}^{*}\right)+\left(c\left(x_{i}\right) u\left(x_{i}\right)-f\left(x_{i}\right), \varphi_{i, \Omega_{i}}^{*}\right) \\
& =\left((b-\bar{b}) u^{\prime}+c\left(u-u\left(x_{i}\right)\right)+\left(c-c\left(x_{i}\right)\right) u\left(x_{i}\right)+\left(f\left(x_{i}\right)-f\right), \varphi_{i, \Omega_{i}}^{*}\right) .
\end{aligned}
$$

Since

$$
\left|u(x)-u\left(x_{i}\right)\right|=\left|\int_{x_{i}}^{x} u^{\prime}(s) d s\right| \leq \int_{x_{i-1}}^{x_{i+1}}\left|u^{\prime}(s)\right| d s=: \mathcal{J}_{i},
$$

it follows, from (4.9), that

$$
\left|\bar{a}\left(u-u_{h}^{*}, \varphi_{i, \Omega_{i}}^{*}\right)\right| \leq C \bar{h}_{i}\left(I_{i}+h_{i}\right) .
$$

Assume for the moment that, for $0<i<N$

$$
\mathcal{J}_{i} \leq C \bar{h}_{i} .
$$

Then using equation (4.6) and inequality (4.10), we get

$$
\left|a\left(u-u_{h}^{*}, v_{h}^{*}\right)\right| \leq C h \sum_{i=1}^{N-1} \bar{h}_{i}^{1 / 2}\left|v_{i}^{*}\right| \bar{h}_{i}^{1 / 2} \leq C h\left\|v_{h}^{*}\right\|_{d},
$$


where we have used the Cauchy-Schwarz inequality for the right hand side of the last inequality. It remains therefore to verify (4.10). It is clear that for $0<i<N$

$$
\begin{aligned}
\mathcal{J}_{i} \leq C \int_{x_{i-1}}^{x_{i+1}}\left(1+\varepsilon^{-1} e^{-\alpha_{i}(1-x) / \varepsilon}\right) d x & =C \bar{h}_{i}+C\left(e^{-\alpha_{i}\left(1-x_{i+1}\right) / \varepsilon}-e^{-\alpha_{i}\left(1-x_{i-1}\right) / \varepsilon}\right) \\
& =C\left(\bar{h}_{i}+\frac{2 \alpha_{i} h_{i}}{\varepsilon} e^{-\alpha_{i}\left(1-x_{i}\right) / \varepsilon}\right)+O\left(h_{i}^{3}\right) .
\end{aligned}
$$

This proves the desired result.

The following lemma from [28] allows us to make a convenient transition from $L_{1}-$ norm to the $L_{2}-$ norm:

Lemma 4.7. Let $V_{h}^{*}$ be the space of discrete $L$-splines. For any $v_{h}^{*} \in V_{h}^{*}$, we have

$$
\left\|v_{h}^{* \prime}\right\|_{L_{1}} \leq C \varepsilon^{1 / 2} h^{-1 / 2}\left\|v_{h}^{* \prime}\right\|_{L_{2}} .
$$

Now we are ready to state the main result.

Theorem 4.8. Let $u$ be the solution of (1.1) and $u_{h}^{*}$ be the solution of (3.7). Then we have,

$$
\left\|u-u_{h}^{*}\right\|_{\varepsilon, d}^{2} \leq C h\left(1+M^{-1 / 2} \ln ^{2}\left(M / h_{i}\right)\right) .
$$

Proof. Since the bilinear form $\bar{a}(\cdot, \cdot)$ is coercive in $H_{0}^{1}(\Omega)$, we have

$$
\beta\left\|u-u_{h}^{*}\right\|_{\varepsilon, d}^{2} \leq \bar{a}\left(u-u_{h}^{*}, u-u_{h}^{*}\right)=\bar{a}\left(u-u_{h}^{*}, u_{I}^{*}-u_{h}^{*}\right)+\bar{a}\left(u-u_{h}^{*}, u-u_{I}^{*}\right) .
$$

For the first term on the right hand side of (4.12), by exploiting Lemma 4.6, we obtain

$$
\begin{aligned}
\bar{a}\left(u-u_{h}^{*}, u_{I}^{*}-u_{h}^{*}\right) & \leq C h\left\|u_{I}^{*}-u_{h}^{*}\right\|_{d} \leq C h\left(\left\|u_{I}^{*}-u\right\|_{\varepsilon, d}+\left\|u-u_{h}^{*}\right\|_{\varepsilon, d}\right) \\
& \leq C h\left\|u_{I}^{*}-u\right\|_{\varepsilon, d}+C\left(\frac{h}{2}+\frac{h}{2}\left\|u-u_{h}^{*}\right\|_{\varepsilon, d}^{2}\right)
\end{aligned}
$$

To estimate the second term on the right hand side of (4.12), observe that

$$
\begin{aligned}
\bar{a}\left(u-u_{h}^{*}, u-u_{I}^{*}\right) & =\varepsilon\left(\left(u-u_{h}^{*}\right)^{\prime},\left(u-u_{I}^{*}\right)^{\prime}\right)+\left(\bar{b}\left(u-u_{h}^{*}\right)^{\prime},\left(u-u_{I}^{*}\right)\right)+\left(c\left(u-u_{h}^{*}\right),\left(u-u_{I}^{*}\right)\right)_{d} \\
& =\text { Diffusion + Convection + Reaction. }
\end{aligned}
$$

For the reaction term, we have

$$
\left(c\left(u-u_{h}^{*}\right),\left(u-u_{I}^{*}\right)\right)_{d}=\sum_{i=1}^{N-1} \bar{h}_{i} c\left(x_{i}\right)\left(u-u_{h}^{*}\right)\left(x_{i}\right)\left(u-u_{I}^{*}\right)\left(x_{i}\right)=0 .
$$

To estimate the diffusion term in (4.14), we employ the Cauchy-Schwarz and Young's inequalities (3.2), successively, to obtain that

$$
\begin{aligned}
\varepsilon\left(\left(u-u_{h}^{*}\right)^{\prime},\left(u-u_{I}^{*}\right)^{\prime}\right) & \leq \varepsilon^{1 / 2}\left\|\left(u-u_{h}^{*}\right)^{\prime}\right\|_{L_{2}} \varepsilon^{1 / 2}\left\|\left(u-u_{I}^{*}\right)^{\prime}\right\|_{L_{2}} \\
& \leq \gamma_{1} \varepsilon\left\|\left(u-u_{h}^{*}\right)^{\prime}\right\|_{L_{2}}^{2}+C\left(\gamma_{1}\right) \varepsilon\left\|\left(u-u_{I}^{*}\right)^{\prime}\right\|_{L_{2}}^{2} \\
& \leq \gamma_{1}\left\|\left(u-u_{h}^{*}\right)\right\|_{\varepsilon, d}^{2}+C\left(\gamma_{1}\right)\left\|\left(u-u_{I}^{*}\right)\right\|_{\varepsilon, d}^{2} .
\end{aligned}
$$


Here, the constant $\gamma_{1}$ will be chosen later. Now, we are moving on to the convection term in (4.14). Since

$$
\begin{aligned}
\left(\bar{b}\left(u-u_{I}^{*}\right)^{\prime},\left(u-u_{I}^{*}\right)\right) & =\sum_{i=1}^{N} \int_{x_{i-1}}^{x_{i}} \bar{b}\left(u-u_{I}^{*}\right)^{\prime}\left(u-u_{I}^{*}\right) d x=\frac{1}{2} \sum_{i=1}^{N} \bar{b} \int_{x_{i-1}}^{x_{i}} \frac{d}{d x}\left(u-u_{I}^{*}\right)^{2} d x \\
& =\frac{1}{2} \sum_{i=1}^{N} \bar{b}\left(\left(u-u_{I}^{*}\right)^{2}\left(x_{i}\right)-\left(u-u_{I}^{*}\right)^{2}\left(x_{i-1}\right)\right) \\
& =\frac{1}{2} \sum_{i=1}^{N} \bar{b}\left(\left(u-u_{I}^{*}\right)\left(x_{i}\right)\left(u-u_{I}^{*}\right)\left(x_{i}\right)-\left(u-u_{I}^{*}\right)\left(x_{i-1}\right)\left(u-u_{I}^{*}\right)\left(x_{i-1}\right)\right) \\
& =0,
\end{aligned}
$$

we get

$$
\left|\left(\bar{b}\left(u-u_{h}^{*}\right)^{\prime},\left(u-u_{I}^{*}\right)\right)\right|=\left|\left(\bar{b}\left(u_{I}^{*}-u_{h}^{*}\right)^{\prime},\left(u-u_{I}^{*}\right)\right)\right| \leq C|| u-u_{I}^{*}\left\|_{\infty}\right\|\left(u_{I}^{*}-u_{h}^{*}\right)^{\prime} \|_{L_{1}} .
$$

Applying Lemma 4.7 to the last term in the above inequality, we get

$$
\begin{aligned}
& \left|\left(\bar{b}\left(u-u_{h}^{*}\right)^{\prime},\left(u-u_{I}^{*}\right)\right)\right| \leq C \varepsilon^{1 / 2} h^{-1 / 2}\left\|u-u_{I}^{*}\right\|_{\infty}\left\|\left(u_{I}^{*}-u_{h}^{*}\right)^{\prime}\right\|_{L_{2}} \\
& \leq C \varepsilon^{1 / 2} h^{-1 / 2}\left\|u-u_{I}^{*}\right\|_{\infty}\left[\left\|\left(u_{I}^{*}-u\right)^{\prime}\right\|_{L_{2}}+\left\|\left(u-u_{h}^{*}\right)^{\prime}\right\|_{L_{2}}\right] \\
& =C \varepsilon^{1 / 2} h^{-1 / 2}\left\|u-u_{I}^{*}\right\|_{\infty}\left\|\left(u-u_{I}^{*}\right)^{\prime}\right\|_{L_{2}}+C \varepsilon^{1 / 2} h^{-1 / 2}\left\|u-u_{I}^{*}\right\|_{\infty}\left\|\left(u-u_{h}^{*}\right)^{\prime}\right\|_{L_{2}} \\
& \leq C h^{-1 / 2}\left\|u-u_{I}^{*}\right\|_{\infty}\left\|\left(u-u_{I}^{*}\right)\right\|_{\varepsilon, d}+C \varepsilon^{1 / 2} h^{-1 / 2}\left\|u-u_{I}^{*}\right\|_{\infty}\left\|\left(u-u_{h}^{*}\right)^{\prime}\right\|_{L_{2}} \\
& \leq C h^{-1 / 2}\left\|u-u_{I}^{*}\right\|_{\infty}\left\|\left(u-u_{I}^{*}\right)\right\|_{\varepsilon, d}+C\left(\gamma_{2}\right) h^{-1}\left\|u-u_{I}^{*}\right\|_{\infty}^{2}+\gamma_{2} \varepsilon\left\|\left(u-u_{h}^{*}\right)^{\prime}\right\|_{L_{2}}^{2} \\
& \leq C h^{-1 / 2}\left\|u-u_{I}^{*}\right\|_{\infty}\left\|\left(u-u_{I}^{*}\right)\right\|_{\varepsilon, d}+C\left(\gamma_{2}\right) h^{-1}\left\|u-u_{I}^{*}\right\|_{\infty}^{2}+\gamma_{2}\left\|\left(u-u_{h}^{*}\right)\right\|_{\varepsilon, d}^{2},
\end{aligned}
$$

where we have used the triangle inequality, the definition of discrete energy norm $\|\cdot\|_{\varepsilon, d}$ and Young's inequality (3.2) at the second, fourth and fifth lines, respectively. By substituting equations (4.15)-(4.17) into equation (4.14) and then substituting equations (4.13)-(4.14) into equation (4.12) we get,

$$
\begin{aligned}
\left(\beta-\gamma_{1}-\gamma_{2}-C \frac{h}{2}\right)\left\|u-u_{h}^{*}\right\|_{\varepsilon, d}^{2} & \leq C\left(\gamma_{1}\right)\left\|\left(u-u_{I}^{*}\right)\right\|_{\varepsilon, d}^{2}+C h^{-1 / 2}\left\|u-u_{I}^{*}\right\|_{\infty}\left\|u-u_{I}^{*}\right\|_{\varepsilon, d} \\
& +C\left(\gamma_{2}\right) h^{-1}\left\|u-u_{I}^{*}\right\|_{\infty}^{2}+C h\left\|u-u_{I}^{*}\right\|_{\varepsilon, d}+C \frac{h}{2},
\end{aligned}
$$

where $C\left(\gamma_{i}\right)=1 /\left(4 \gamma_{i}\right), i=1,2$. The result follows by recalling Lemma 4.4 and Lemma 4.5.

We note that the error bound given in (4.11) is consistent with the bound of the numerical method using $L$-splines and one can recover the optimal convergence rates in (3.5) as the local mesh is refined.

\section{Conclusion}

The convergence properties of a numerical method in the context of standard Galerkin FEM for the numerical solution of singularly perturbed two-point boundary-value problems on piecewise-uniform meshes is investigated. Recently, it is quite a common practice 
that the polynomial finite element spaces are enriched by appropriate smooth functions to get improved approximations. However, the computer implementation of such approaches use their discrete counterparts. Here, the proposed numerical algorithm uses the discrete $L$-spline basis functions in place of their continuous counterparts, for which we tried to reveal how much error the use of discrete $L$-spline basis functions contributes to the overall numerical method. Consequently, the classical convergence rates with some modification representing the effect of the local discretization can be recovered.

Acknowledgment. The authors express their sincere thanks to the referees whose valuable comments helped to improve the presentation.

\section{References}

[1] F. Brezzi, P. Houston, D. Marini and E. Süli, Modeling subgrid viscosity for advectiondiffusion problems, Comput. Meth. Appl. Mech. Eng. 190, 1601-1610, 2000.

[2] F. Brezzi, T.J.R. Hughes, L.D. Marini, A. Russo and E. Süli, A priori error analysis of residual-free bubbles for advection-diffusion problems, SIAM J. Numer. Anal. 36 (6), 1933-1948, 1999.

[3] F. Brezzi, D. Marini and A. Russo, Applications of pseudo residual-free bubbles to the stabilization of convection-diffusion problems, Comput. Meth. Appl. Mech. Eng. 166, 51-63, 1998.

[4] F. Brezzi and A. Russo, Choosing bubbles for advection-diffusion problems, Math. Models Meth. Appl. Sci. 4, 571-587, 1994.

[5] A.N. Brooks and T.J.R. Hughes, Streamline upwind/Petrov-Galerkin formulations for convection-dominated flows with particular emphasis on the incompressible NavierStokes equations, Comput. Meth. Appl. Mech. Eng. 32, 199-259, 1982.

[6] A. Buffa, T.J.R. Hughes and G. Sangalli, Analysis of a Multiscale Discontinuous Galerkin Method for Convection Diffusion Problems, SIAM J. Numer. Anal. 44, 1420$1440,2006$.

[7] A. Cangiani and E. Süli, Enhanced residual-free bubble method for convection-diffusion problems, Inter. J. Numer. Methods Fluids 47, 1307-1313, 2005.

[8] B. Cockburn, Discontinuous Galerkin methods for convection-dominated problems, Springer Berlin Heidelberg, 1999.

[9] L.P. Franca, S.L. Frey and T.J.R. Hughes, Stabilized finite element methods: I. Application to the advective-diffusive model, Comput. Meth. Appl. Mech. Eng. 95, 253-276, 1992.

[10] L.P. Franca and F.N. Hwang, Refining the submesh strategy in the two-level finite element method: application to the advection diffusion equation, Int. J. Numer. Methods Fluids 39, 161-187, 2002.

[11] L.P. Franca, A.L. Madureira and F. Valentin, Towards multiscale functions: enriching finite element spaces with local but not bubble-like functions, Comput. Meth. Appl. Mech. Eng. 194, 3006-3021, 2005.

[12] L.P. Franca and A. Nesliturk, On a two-level finite element method for the incompressible Navier-Stokes equations, Int. J. Numer. Methods Fluids 52, 433-453, 2001.

[13] L.P. Franca, A. Nesliturk and M. Stynes, On the stability of residual-free bubbles for convection-diffusion problems and their approximation by a two-level finite element method, Comput. Meth. Appl. Mech. Eng. 166, 35-49, 1998.

[14] L.P. Franca, J.V.A. Ramalho and F. Valentin, Multiscale and residual-free bubble functions for reaction-advection-diffusion problems, Int. J. Multiscale Com. 3, 297$312,2005$.

[15] L.P. Franca and L. Tobiska, Stability of the residual free bubble method for bilinear finite elements on rectangular grids, IMA J. Numer. Anal. 22, 73-87, 2002. 
[16] E. C. Gartland, Graded-mesh difference schemes for singularly perturbed two-point boundary-value problems, Math. Comp. 51, 631-657, 1988.

[17] T.J.R. Hughes, Multiscale phenomena: Green's functions, the Dirichlet-to-Neumann formulation, subgrid scale models, bubbles and the origin of stabilized methods, Comput. Meth. Appl. Mech. Eng. 127, 387-401, 1995.

[18] T.J.R. Hughes, G.R. Feijoo, L. Mazzei and J.B. Quincy, The variational multiscale method - a paradigm for computational mechanics, Comput. Meth. Appl. Mech. Eng. 166 (1-2), 3-24, 1998.

[19] R.B. Kellog and A. Tsan, Analysis of some difference approximations for a singular perturbation problem without turning points, Math. Comp. 32, 1025-1039, 1978.

[20] N. Kopteva and E. O'Riordan, Shishkin meshes in the numerical solution of singularly perturbed differential equations, Int. J. Numer. Anal. Model. 7, 393-415, 2010.

[21] T. Linss, The necessity of Shishkin-decompositions, Appl. Math. Lett. 14, 891-896, 2001.

[22] J.J.H. Miller, E. O'Riordan and G.I. Shishkin, Fitted Numerical Methods For Singular Perturbation Problems, Singapore, New Jersey, London, Hong Kong: World Scientific, 1996.

[23] E. O'Riordan and M. Stynes, A globally uniformly convergent finite element method for a singularly perturbed elliptic problem in two dimensions, Math. Comp. 57, 47-62, 1991.

[24] H.G. Roos , M. Stynes and L. Tobiska, Robust Numerical Methods For Singularly Perturbed Differential Equations, Verlag, Berlin: Springer, 2008.

[25] A. Sendur and A. Nesliturk, Applications of the pseudo residual-free bubbles to the stabilization of convection-diffusion-reaction problems, Calcolo 49, 1-19, 2012.

[26] G.I. Shishkin, Grid approximation of singularly perturbed parabolic equations with internal layers, Soviet J. Numer. Anal. Math. Appl. Modelling. 3, 393-407, 1988.

[27] M. Stynes, Steady-state convection-diffusion problems, Acta Numer. 14, 445-508, 2005.

[28] M. Stynes and E. O'Riordan, An analysis of a singularly perturbed two-point boundary value problem using only finite element techniques, Math. Comp. 56, 663-675, 1991.

[29] M. Stynes and E. O'Riordan, A uniformly convergent Galerkin method on a Shishkin mesh for a convection-diffusion problem, J. Math. Anal. Appl. 214, 36-54, 1997.

[30] G. Sun and M. Stynes, Finite element methods for singularly perturbed high order elliptic two-point boundary-value problems. II: Convection-diffusion type problems, IMA. J. of Numer. Anal. 15, 197-219, 1995.

\section{Appendix A.}

The theoretical results described here are based on the techniques presented in $[22,28$, 30]. Recall that we wish to solve (3.3) in $\Omega_{i}$, that is,

$$
\left\{\begin{array}{l}
-\varepsilon \varphi_{i, \Omega_{i}}^{\prime \prime}+\bar{b} \varphi_{i, \Omega_{i}}^{\prime}+\bar{c} \varphi_{i, \Omega_{i}}=0, \\
\varphi_{i, \Omega_{i}}\left(x_{i-1}\right)=0, \quad \varphi_{i, \Omega_{i}}\left(x_{i}\right)=1 .
\end{array}\right.
$$

We solve $\varphi_{i}$ in $\Omega_{i+1}$ in the same manner and obtain qualitatively similar estimates. Therefore, we skip the proof for $\Omega_{i+1}$. The following lemma gives information about the derivatives of $\varphi_{i, \Omega_{i}}$.

Lemma A.1. Let $\varphi_{i, \Omega_{i}}$ be the solution of (A.1). Then, for all $x \in \bar{\Omega}_{i}$, we have

$$
\left|\varphi_{i, \Omega_{i}}^{(m)}(x)\right| \leq C\left(1+\varepsilon^{-m} h_{i}^{m} e^{-\frac{\alpha_{i}}{\varepsilon}\left(x_{i}-x\right)}\right) \quad 0 \leq m \leq 3 .
$$

Proof. The estimate is obtained by following the steps given in [22]. 
However, decomposing $\varphi_{i, \Omega_{i}}$ into the smooth and singular components, we may obtain more refined bounds on the derivatives. They are given in the following lemma.

Lemma A.2. The solution $\varphi_{i, \Omega_{i}}$ of (A.1) has the following decomposition

$$
\varphi_{i, \Omega_{i}}=v_{i, \Omega_{i}}+w_{i, \Omega_{i}} .
$$

Then, for all $x \in \bar{\Omega}_{i}$, the smooth and singular components satisfy the following bounds, respectively.

$$
\begin{gathered}
\left|v_{i, \Omega_{i}}^{(m)}(x)\right| \leq C, \quad 0 \leq m \leq 3, \\
\left|w_{i, \Omega_{i}}^{(m)}(x)\right| \leq C, h_{i}^{m} \varepsilon^{-m} e^{-\frac{\alpha_{i}}{\varepsilon}\left(x_{i}-x\right)} \quad 0 \leq m \leq 3 .
\end{gathered}
$$

Proof. The estimate can be obtained by following the method of proof given in [22].

Before moving on to the main estimates, some bounds for the interpolation error in the finite element spaces are required. The finite element trial and test spaces that we wish to work are the space of continuous piecewise linear polynomials on the piecewise-uniform mesh $\Omega_{i, \tau_{i}}^{M}$ which are defined by

$$
\begin{aligned}
& W_{i}^{*}\left(\Omega_{i, \tau_{i}}^{M}\right) \\
& \quad=\left\{\psi_{i}^{*} \in H^{1}\left(\Omega_{i}\right):\left.\psi_{i}^{*}\right|_{\Omega_{i, k}^{*}} \in P_{1}\left(\Omega_{i, k}^{*}\right) \text { with } \Omega_{i, k}^{*} \subset \Omega_{i} \text { and } \psi_{i}^{*}\left(x_{i-1}\right)=0, \psi_{i}^{*}\left(x_{i}\right)=1\right\},
\end{aligned}
$$

and

$$
W_{i, 0}^{*}\left(\Omega_{i, \tau_{i}}^{M}\right)=\left\{\psi_{i}^{*} \in H_{0}^{1}\left(\Omega_{i}\right):\left.\psi_{i}^{*}\right|_{\Omega_{i, k}^{*}} \in P_{1}\left(\Omega_{i, k}^{*}\right) \text { with } \Omega_{i, k}^{*} \subset \Omega_{i}\right\},
$$

respectively, where $\Omega_{i, k}^{*}=\left(x_{i, k-1}^{*}, x_{i, k}^{*}\right)$ and $P_{1}\left(\Omega_{i, k}^{*}\right)$ is the space of linear functions on $\Omega_{i, k}^{*}$.

\section{A.1. Interpolation estimates at element level}

The following lemma provides an $\varepsilon$-uniform estimate of $\left(\bar{\varphi}_{i, \Omega_{i}}-\varphi_{i, \Omega_{i}}\right)$ in the maximum norm, where $\bar{\varphi}_{i, \Omega_{i}}$ is the piecewise linear $W_{i}^{*}\left(\Omega_{i, \tau_{i}}^{M}\right)$-interpolant of $\varphi_{i, \Omega_{i}}$. In the proofs of the following lemmas and theorems, we make intense use of the arguments given in [21,22].

Lemma A.3. Let $\bar{\varphi}_{i, \Omega_{i}}$ denote the $W_{i}^{*}\left(\Omega_{i, \tau_{i}}^{M}\right)$-interpolant of the exact solution $\varphi_{i, \Omega_{i}}$ of (A.1). Then, we have

$$
\left\|\bar{\varphi}_{i, \Omega_{i}}-\varphi_{i, \Omega_{i}}\right\|_{\infty, \Omega_{i}} \leq h_{i}^{2} \bar{\gamma}_{i, \infty}(M), \quad \text { where } \bar{\gamma}_{i, \infty}(M)=C M^{-2}\left(\ln \left(M / h_{i}\right)\right)^{2} .
$$

Proof. The bound can be obtained on both sides of subinterval $\Omega_{i, k}^{*}$ separately. We recall that for any function $g \in W_{i}^{*}\left(\Omega_{i, \tau_{i}}^{M}\right)$

$$
\bar{g}=g\left(x_{i, k-1}^{*}\right) \phi_{i, k-1}^{*}+g\left(x_{i, k}^{*}\right) \phi_{i, k}^{*} \quad \text { in } \Omega_{i, k}^{*},
$$

where $\left\{\phi_{i, k}^{*}\right\}_{k=1}^{M-1}$ denotes the standard piecewise linear basis functions on $\Omega_{i, \tau_{i}}^{M}$. So it is obvious that

$$
|\bar{g}| \leq \max _{\Omega_{i, k}^{*}}|g(x)| .
$$

Furthermore, let us recall the error bound in the Taylor's expansion, i.e.,

$$
|g-\bar{g}| \leq C h_{k}^{* 2} \max _{\Omega_{i, k}^{*}}\left|g^{\prime \prime}(x)\right| .
$$

From (A.4) and Lemma A.1, on $\Omega_{i, k}^{*}$, we have

$$
\left|\bar{\varphi}_{i, \Omega_{i}}-\varphi_{i, \Omega_{i}}\right| \leq C h_{k}^{* 2} \max _{\Omega_{i, k}^{*}}\left|\varphi_{i, \Omega_{i}}^{\prime \prime}(x)\right| \leq C h_{k}^{* 2} \max _{\Omega_{i, k}^{*}}\left|1+\frac{h_{i}^{2}}{\varepsilon^{2}} e^{-\frac{\alpha_{i}}{\varepsilon}\left(x_{i}-x\right)}\right| .
$$


Using Lemma A.2, (A.3) and (A.4), we also have

$$
\begin{aligned}
\left|\bar{\varphi}_{i, \Omega_{i}}-\varphi_{i, \Omega_{i}}\right| & =\left|\bar{v}_{i, \Omega_{i}}+\bar{w}_{i, \Omega_{i}}-v_{i, \Omega_{i}}-w_{i, \Omega_{i}}\right| \leq\left|\bar{v}_{i, \Omega_{i}}-v_{i, \Omega_{i}}\right|+\left|\bar{w}_{i, \Omega_{i}}\right|+\left|w_{i, \Omega_{i}}\right| \\
& \leq C h_{k}^{* 2} \max _{\Omega_{i, k}^{*}}\left|v_{i, \Omega_{i}}^{\prime \prime}(x)\right|+2 \max _{\Omega_{i, k}^{*}}\left|w_{i, \Omega_{i}}\right| \\
& \leq C h_{k}^{* 2}+2 \max _{\Omega_{i, k}^{*}}\left|e^{-\frac{\alpha_{i}}{\varepsilon}\left(x_{i}-x\right)}\right| .
\end{aligned}
$$

We will make use of both (A.5) and (A.6) in different places, whichever is appropriate. We shall assume that $\tau_{i}=\frac{2 \varepsilon}{\alpha_{i}} \ln \left(M / h_{i}\right)$, otherwise $\varepsilon \geq \frac{\alpha_{i} h_{i}}{4 \ln \left(M / h_{i}\right)}$, in which case $\varepsilon$ is not so small and therefore the problem can be analyzed in the classical way. That is, we will consider the case

$$
\tau_{i}=\frac{2 \varepsilon \ln \left(M / h_{i}\right)}{\alpha_{i}}<\frac{h_{i}}{2} .
$$

Now suppose that $k$ satisfies $\frac{M}{2}<k \leq M$ which contains a neighborhood of the boundary layer. Then $h_{k}^{*}=\frac{2 \tau_{i}}{M}$ and therefore

$$
h_{k}^{*}=\frac{2 \tau_{i}}{M} \leq h_{i} M^{-1},
$$

for sufficiently small $\varepsilon$. Moreover, we have,

$$
\frac{h_{k}^{*} h_{i}}{\varepsilon} e^{-\frac{\alpha_{i}}{2 \varepsilon}\left(x_{i}-x\right)}=\frac{2 \tau_{i}}{M} \frac{h_{i}}{\varepsilon} e^{-\frac{\alpha_{i}}{2 \varepsilon}\left(x_{i}-x\right)}=\frac{2 \varepsilon}{\alpha_{i}} \ln \left(M / h_{i}\right) \frac{2}{M} \frac{h_{i}}{\varepsilon} e^{-\frac{\alpha_{i}}{2 \varepsilon}\left(x_{i}-x\right)} \leq C h_{i} M^{-1} \ln \left(M / h_{i}\right) .
$$

The result follows by combining these two inequalities with (A.5), i.e.,

$$
\left|\bar{\varphi}_{i, \Omega_{i}}-\varphi_{i, \Omega_{i}}\right| \leq C h_{i}^{2} M^{-2}\left(\ln \left(M / h_{i}\right)\right)^{2} .
$$

Now consider the coarse mesh points for $1 \leq k \leq \frac{M}{2}$, which describe the outside of the boundary layer, then we have

$$
h_{k}^{*}=\frac{2\left(h_{i}-\tau_{i}\right)}{M} \leq C h_{i} M^{-1} .
$$

Moreover, we have,

$$
e^{-\frac{\alpha_{i}}{\varepsilon}\left(x_{i}-x\right)} \leq e^{-\frac{\alpha_{i}}{\varepsilon} \tau_{i}}=h_{i}^{2} M^{-2},
$$

for sufficiently small $\varepsilon$. Here we have used $x-x_{i} \geq \tau_{i}$. The result follows by combining (A.7) and (A.8) with (A.6).

The following lemma provides an $\varepsilon$-uniform estimate of $\left(\bar{\varphi}_{i, \Omega_{i}}-\varphi_{i, \Omega_{i}}\right)$ in the energy norm.

Lemma A.4. Let $\bar{\varphi}_{i, \Omega_{i}}$ be the $W_{i}^{*}\left(\Omega_{i, \tau_{i}}^{M}\right)$-interpolant of the exact solution $\varphi_{i, \Omega_{i}}$ of (A.1). Then, we have

$$
\left\|\bar{\varphi}_{i, \Omega_{i}}-\varphi_{i, \Omega_{i}}\right\|_{\varepsilon, \Omega_{i}} \leq h_{i} \sqrt{\bar{\gamma}_{i, \infty}(M)} .
$$

Proof. Using the definition of $\varepsilon$-weighted norm, we have

$\left\|\bar{\varphi}_{i, \Omega_{i}}-\varphi_{i, \Omega_{i}}\right\|_{\varepsilon, \Omega_{i}}^{2}=\varepsilon\left(\left(\bar{\varphi}_{i, \Omega_{i}}-\varphi_{i, \Omega_{i}}\right)^{\prime},\left(\bar{\varphi}_{i, \Omega_{i}}-\varphi_{i, \Omega_{i}}\right)^{\prime}\right)_{\Omega_{i}}+\left(\bar{\varphi}_{i, \Omega_{i}}-\varphi_{i, \Omega_{i}}, \bar{\varphi}_{i, \Omega_{i}}-\varphi_{i, \Omega_{i}}\right)_{\Omega_{i}}$.

Each term on the right hand side is considered separately. We start the calculations with the second term, that is,

$$
\left(\bar{\varphi}_{i, \Omega_{i}}-\varphi_{i, \Omega_{i}}, \bar{\varphi}_{i, \Omega_{i}}-\varphi_{i, \Omega_{i}}\right)_{\Omega_{i}} \leq h_{i}\left\|\bar{\varphi}_{i, \Omega_{i}}-\varphi_{i, \Omega_{i}}\right\|_{\infty, \Omega_{i}}^{2} .
$$


The first term can be bounded by using integration by parts and recalling that $\left(\bar{\varphi}_{i, \Omega_{i}}-\right.$ $\left.\varphi_{i, \Omega_{i}}\right)\left(x_{i, k}^{*}\right)=0$ for each $k, 0 \leq k \leq M$,

$$
\begin{aligned}
\varepsilon\left(\left(\bar{\varphi}_{i, \Omega_{i}}-\varphi_{i, \Omega_{i}}\right)^{\prime},\left(\bar{\varphi}_{i, \Omega_{i}}-\varphi_{i, \Omega_{i}}\right)^{\prime}\right)_{\Omega_{i}} & =\varepsilon \sum_{k=1}^{M} \int_{x_{i, k-1}^{*}}^{x_{i, k}^{*}}\left[\left(\bar{\varphi}_{i, \Omega_{i}}-\varphi_{i, \Omega_{i}}\right)^{\prime}\right]^{2} d x \\
& =-\varepsilon \sum_{k=1}^{M} \int_{x_{i, k-1}^{*}}^{x_{i, k}^{*}}\left(\bar{\varphi}_{i, \Omega_{i}}-\varphi_{i, \Omega_{i}}\right)^{\prime \prime}\left(\bar{\varphi}_{i, \Omega_{i}}-\varphi_{i, \Omega_{i}}\right) d x \\
& =\varepsilon\left(\varphi_{i, \Omega_{i}}^{\prime \prime}, \bar{\varphi}_{i, \Omega_{i}}-\varphi_{i, \Omega_{i}}\right)_{\Omega_{i}} .
\end{aligned}
$$

Here, we have used $\bar{\varphi}_{i, \Omega_{i}}^{\prime \prime}=0$ on each $\Omega_{i, k}^{*}$. The estimate for the second derivative of $\varphi_{i, \Omega_{i}}$ in Lemma A.1 yields

$$
\left|\varepsilon\left(\varphi_{i, \Omega_{i}}^{\prime \prime}, \bar{\varphi}_{i, \Omega_{i}}-\varphi_{i, \Omega_{i}}\right)_{\Omega_{i}}\right| \leq\left\|\bar{\varphi}_{i, \Omega_{i}}-\varphi_{i, \Omega_{i}}\right\|_{\infty, \Omega_{i}} \int_{x_{i-1}}^{x_{i}} \varepsilon\left|\varphi_{i, \Omega_{i}}^{\prime \prime}\right| d x \leq\left\|\bar{\varphi}_{i, \Omega_{i}}-\varphi_{i, \Omega_{i}}\right\|_{\infty, \Omega_{i}} .
$$

Combining (A.10) and (A.11) leads to

$$
\begin{aligned}
\left\|\bar{\varphi}_{i, \Omega_{i}}-\varphi_{i, \Omega_{i}}\right\|_{\varepsilon, \Omega_{i}}^{2} & \leq h_{i}\left\|\bar{\varphi}_{i, \Omega_{i}}-\varphi_{i, \Omega_{i}}\right\|_{\infty, \Omega_{i}}^{2}+\left\|\bar{\varphi}_{i, \Omega_{i}}-\varphi_{i, \Omega_{i}}\right\|_{\infty, \Omega_{i}} \\
& \leq\left\|\bar{\varphi}_{i, \Omega_{i}}-\varphi_{i, \Omega_{i}}\right\|_{\infty, \Omega_{i}}\left(1+h_{i}\left\|\bar{\varphi}_{i, \Omega_{i}}-\varphi_{i, \Omega_{i}}\right\|_{\infty, \Omega_{i}}\right) .
\end{aligned}
$$

The proof is completed by applying the bound in (A.2).

\section{A.2. Approximation method for basis functions}

Let us recall the weak form of the problem (A.1):

$$
\left\{\begin{array}{l}
\text { Find } \varphi_{i, \Omega_{i}} \in H^{1}\left(\Omega_{i}\right), \text { such that } \\
a^{*}\left(\varphi_{i, \Omega_{i}}, \psi_{i}\right)=0, \quad \forall \psi_{i} \in H_{0}^{1}\left(\Omega_{i}\right),
\end{array}\right.
$$

where $a^{*}(\cdot, \cdot)$ is given by

$$
a^{*}(u, v)=\varepsilon\left(u^{\prime}, v^{\prime}\right)_{\Omega_{i}}+\left(\bar{b} u^{\prime}, v\right)_{\Omega_{i}}+(\bar{c} u, v)_{\Omega_{i}} .
$$

The standard Galerkin approximation of problem (A.12) on $\Omega_{i, \tau_{i}}^{M}$ reads:

$$
\left\{\begin{array}{l}
\text { Find } \varphi_{i, \Omega_{i}}^{*} \in W_{i}^{*}\left(\Omega_{i, \tau_{i}}^{M}\right), \text { such that } \\
a^{*}\left(\varphi_{i, \Omega_{i}}^{*}, \psi_{i}^{*}\right)=0 \quad \forall \psi_{i}^{*} \in W_{i, 0}^{*}\left(\Omega_{i, \tau_{i}}^{M}\right) .
\end{array}\right.
$$

\section{A.3. Convergence analysis}

Here, we shall obtain the error estimates between $L$-spline basis functions $\varphi_{i}$ and its finite element approximation $\varphi_{i}^{*}$. Before proving these estimates, we need some preliminary results.

A.3.1. Preliminary results. For the convergence analysis, we will first show that $a^{*}(\cdot, \cdot)$ is $\varepsilon$-uniformly coercive on $W_{i, 0}^{*}\left(\Omega_{i, \tau_{i}}^{M}\right)$ with respect to $\|\cdot\|_{\varepsilon, \Omega_{i}}$, which is given in the next lemma.

Lemma A.5. For any function $\psi_{i}^{*} \in W_{i, 0}^{*}\left(\Omega_{i, \tau_{i}}^{M}\right)$, we have

$$
a^{*}\left(\psi_{i}^{*}, \psi_{i}^{*}\right) \geq \beta_{i}\left\|\psi_{i}^{*}\right\|_{\varepsilon, \Omega_{i}}^{2} .
$$


Proof. From the definition of the bilinear form, for any $\psi_{i}^{*} \in W_{i, 0}^{*}\left(\Omega_{i, \tau_{i}}^{M}\right)$, we have

$$
a^{*}\left(\psi_{i}^{*}, \psi_{i}^{*}\right)=\varepsilon\left(\psi_{i}^{* \prime}, \psi_{i}^{* \prime}\right)_{\Omega_{i}}+\left(\bar{b} \psi^{* \prime}, \psi_{i}^{*}\right)_{\Omega_{i}}+\left(\bar{c} \psi_{i}^{*}, \psi_{i}^{*}\right)_{\Omega_{i}} .
$$

But the second term on the right hand side is

$$
\left(\bar{b} \psi^{* \prime}, \psi_{i}^{*}\right)_{\Omega_{i}}=\bar{b} \int_{x_{i-1}}^{x_{i}} \psi^{* \prime} \psi_{i}^{*} d x=\frac{\bar{b}}{2}\left(\psi_{i}^{* 2}\left(x_{i}\right)-\psi_{i}^{* 2}\left(x_{i-1}\right)\right)=0 .
$$

Thus, there exists a positive constant $\beta_{i}=\min \{\bar{c}, 1\}$, which is not depending on the parameter $\varepsilon$, such that

$$
a^{*}\left(\psi_{i}^{*}, \psi_{i}^{*}\right) \geq \beta_{i}\left\|\psi_{i}^{*}\right\|_{\varepsilon, \Omega_{i}}^{2} .
$$

We further need next two lemmas. The first of these is about the Galerkin orthogonality:

Lemma A.6 (Galerkin Orthogonality). Let $\varphi_{i, \Omega_{i}}$ be the solution of (A.1) and $\varphi_{i, \Omega_{i}}^{*}$ be the solution of (A.14). Suppose that $\psi_{i}^{*} \in W_{i}^{*}\left(\Omega_{i, \tau_{i}}^{M}\right)$. Then we have

$$
a^{*}\left(\varphi_{i, \Omega_{i}}^{*}-\varphi_{i, \Omega_{i}}, \psi_{i}^{*}\right)=0 .
$$

Proof. From (A.12) and (A.14) we can obtain the Galerkin orthogonality property.

Lemma A.7. Let $\varphi_{i, \Omega_{i}}$ be the solution of (A.1) and $\varphi_{i, \Omega_{i}}^{*}$ be the solution of (A.14). For any $\psi_{i}^{*} \in W_{i}^{*}\left(\Omega_{i, \tau_{i}}^{M}\right)$, we have

$$
a^{*}\left(\bar{\varphi}_{i, \Omega_{i}}-\varphi_{i, \Omega_{i}}, \psi_{i}^{*}\right) \leq h_{i}^{3 / 2} M \bar{\gamma}_{i, \infty}(M)\left\|\psi_{i}^{*}\right\|_{\varepsilon, \Omega_{i}} .
$$

Proof. From (A.13), we have

$$
\begin{aligned}
a^{*}\left(\bar{\varphi}_{i, \Omega_{i}}-\varphi_{i, \Omega_{i}}, \psi_{i}^{*}\right)= & \varepsilon\left(\left(\bar{\varphi}_{i, \Omega_{i}}-\varphi_{i, \Omega_{i}}\right)^{\prime}, \psi_{i}^{* \prime}\right)_{\Omega_{i}}+\left(\bar{b}\left(\bar{\varphi}_{i, \Omega_{i}}-\varphi_{i, \Omega_{i}}\right)^{\prime}, \psi_{i}^{*}\right)_{\Omega_{i}} \\
& +\left(\bar{c}\left(\bar{\varphi}_{i, \Omega_{i}}-\varphi_{i, \Omega_{i}}\right), \psi_{i}^{*}\right)_{\Omega_{i}} .
\end{aligned}
$$

Integrating by parts and using the facts that

$$
\bar{\varphi}_{i, \Omega_{i}}\left(x_{i, k}^{*}\right)=\varphi_{i, \Omega_{i}}\left(x_{i, k}^{*}\right) \text { and } \psi_{i}^{* \prime \prime}=0 \quad \text { on each } \Omega_{i, k}^{*} .
$$

It is easy to see that

$$
\begin{aligned}
\left(\left(\bar{\varphi}_{i, \Omega_{i}}-\varphi_{i, \Omega_{i}}\right)^{\prime}, \psi_{i}^{* \prime}\right)_{\Omega_{i}} & =0 \\
\left(\bar{b}\left(\bar{\varphi}_{i, \Omega_{i}}-\varphi_{i, \Omega_{i}}\right)^{\prime}, \psi_{i}^{*}\right)_{\Omega_{i, k}^{*}} & =-\left(\bar{b}\left(\bar{\varphi}_{i, \Omega_{i}}-\varphi_{i, \Omega_{i}}\right), \psi_{i}^{* \prime}\right)_{\Omega_{i, k}^{*}} \\
\left(\bar{c}\left(\bar{\varphi}_{i, \Omega_{i}}-\varphi_{i, \Omega_{i}}\right), \psi_{i}^{*}\right)_{\Omega_{i}} & \leq C h_{i}^{1 / 2}\left\|\bar{\varphi}_{i, \Omega_{i}}-\varphi_{i, \Omega_{i}}\right\|_{\infty, \Omega_{i}}\left\|\psi_{i}^{*}\right\|_{L_{2}, \Omega_{i}} .
\end{aligned}
$$

Therefore, we have

$$
\begin{aligned}
\left|\left(\bar{b}\left(\bar{\varphi}_{i, \Omega_{i}}-\varphi_{i, \Omega_{i}}\right)^{\prime}, \psi_{i}^{*}\right)_{\Omega_{i}}\right| & =\left|\sum_{k=1}^{M}\left(\bar{b}\left(\bar{\varphi}_{i, \Omega_{i}}-\varphi_{i, \Omega_{i}}\right), \psi_{i}^{* \prime}\right)_{\Omega_{i, k}^{*}}\right| \\
& \leq|\bar{b}|\left\|\bar{\varphi}_{i, \Omega_{i}}-\varphi_{i, \Omega_{i}}\right\|_{\infty, \Omega_{i}} \int_{x_{i-1}}^{x_{i}}\left|\psi_{i}^{* \prime}(s)\right| d s .
\end{aligned}
$$

The integral on the right hand side is now divided into two parts

$$
\int_{x_{i-1}}^{x_{i}}\left|\psi_{i}^{* \prime}(s)\right| d s=\int_{x_{i-1}}^{x_{i}-\tau_{i}}\left|\psi_{i}^{* \prime}(s)\right| d s+\int_{x_{i}-\tau_{i}}^{x_{i}}\left|\psi_{i}^{* \prime}(s)\right| d s .
$$


Using the Cauchy-Schwarz inequality in the first integral of the right hand side of the above equation and $h_{k}^{*} \geq h_{i} / M$ for $1 \leq k \leq M / 2$, we get

$$
\begin{aligned}
\int_{x_{i-1}}^{x_{i}-\tau_{i}}\left|\psi_{i}^{* \prime}(s)\right| d s & =\sum_{k=1}^{M / 2}\left|\psi_{i}^{*}\left(x_{i, k}^{*}\right)-\psi_{i}^{*}\left(x_{i, k-1}^{*}\right)\right| \leq 2 \sqrt{\frac{M}{h_{i}}} \sum_{k=1}^{M / 2} \bar{h}_{k}^{* 1 / 2}\left|\psi_{i}^{*}\left(x_{i, k}^{*}\right)\right| \\
& \leq \frac{2 M}{\sqrt{h_{i}}}\left\|\psi_{i}^{*}\right\|_{L_{2}, d, \Omega_{i}} \leq \frac{2 M}{\sqrt{h_{i}}}\left\|\psi_{i}^{*}\right\|_{L_{2}, \Omega_{i}} .
\end{aligned}
$$

Here we used the equivalence of the continuous and discrete $L_{2}$-norms, on the space of $W_{i}^{*}\left(\Omega_{i, \tau_{i}}^{M}\right)$. On the other hand, applying the Cauchy-Schwarz inequality for the second integral in the right hand side of (A.18) yields,

$$
\int_{x_{i}-\tau_{i}}^{x_{i}}\left|\psi_{i}^{* \prime}(s)\right| d s \leq \sqrt{\tau_{i}}\left(\int_{x_{i}-\tau_{i}}^{x_{i}}\left|\psi_{i}^{* \prime}(s)\right|^{2} d s\right)^{1 / 2} \leq \sqrt{\tau_{i}}\left\|\psi_{i}^{* \prime}\right\|_{L_{2}, \Omega_{i}} \leq \sqrt{\frac{\tau_{i}}{\varepsilon}}\left\|\psi_{i}^{*}\right\|_{\varepsilon, \Omega_{i}} .
$$

Combining these two results, we obtain that

$$
\int_{x_{i-1}}^{x_{i}}\left|\psi_{i}^{* \prime}(s)\right| d s \leq \max _{\Omega_{i}}\left\{\frac{2 M}{\sqrt{h_{i}}}, \sqrt{\frac{\tau_{i}}{\varepsilon}}\right\}\left\|\psi_{i}^{*}\right\|_{\varepsilon, \Omega_{i}} .
$$

From (A.17) and (A.18), it follows that

$$
\left|a^{*}\left(\bar{\varphi}_{i, \Omega_{i}}-\varphi_{i, \Omega_{i}}, \psi_{i}^{*}\right)\right| \leq\left(|\bar{b}| \max _{\Omega_{i}}\left\{\frac{2 M}{\sqrt{h_{i}}}, \sqrt{\frac{\tau_{i}}{\varepsilon}}\right\}+|\bar{c}| h_{i}^{1 / 2}\right)\left\|\bar{\varphi}_{i, \Omega_{i}}-\varphi_{i, \Omega_{i}}\right\|_{\infty, \Omega_{i}}\left\|\psi_{i}^{*}\right\|_{\varepsilon, \Omega_{i}}
$$

which proves the desired result.

To get the error bounds, we need the following final estimate between $W_{i}^{*}\left(\Omega_{i, \tau_{i}}^{M}\right)$-interpolant of $\varphi_{i, \Omega_{i}}$ and its finite element approximation.

Lemma A.8. Let $\bar{\varphi}_{i, \Omega_{i}}$ be the $W_{i}^{*}\left(\Omega_{i, \tau_{i}}^{M}\right)$-interpolant of $\varphi_{i, \Omega_{i}}$ and $\varphi_{i, \Omega_{i}}^{*}$ be the solution of (A.14). Then

$$
\left\|\varphi_{i, \Omega_{i}}^{*}-\bar{\varphi}_{i, \Omega_{i}}\right\|_{\varepsilon, \Omega_{i}} \leq h_{i}^{3 / 2} M \bar{\gamma}_{i, \infty}(M) .
$$

Proof. Since $\varphi_{i, \Omega_{i}}^{*}-\bar{\varphi}_{i, \Omega_{i}} \in W_{i, 0}^{*}\left(\Omega_{i, \tau_{i}}^{M}\right)$, using the coercivity of $a^{*}(\cdot, \cdot)$ and the Galerkin orthogonality, we have

$$
\begin{aligned}
& \beta_{i}\left\|\varphi_{i, \Omega_{i}}^{*}-\bar{\varphi}_{i, \Omega_{i}}\right\|_{\varepsilon, \Omega_{i}}^{2} \leq a^{*}\left(\varphi_{i, \Omega_{i}}^{*}-\bar{\varphi}_{i, \Omega_{i}}, \varphi_{i, \Omega_{i}}^{*}-\bar{\varphi}_{i, \Omega_{i}}\right) \\
\leq & a^{*}\left(\varphi_{i, \Omega_{i}}-\bar{\varphi}_{i, \Omega_{i}}, \varphi_{i, \Omega_{i}}^{*}-\bar{\varphi}_{i, \Omega_{i}}\right)+a^{*}\left(\varphi_{i, \Omega_{i}}^{*}-\varphi_{i, \Omega_{i}}, \varphi_{i, \Omega_{i}}^{*}-\bar{\varphi}_{i, \Omega_{i}}\right) \\
\leq & \left(\max _{\Omega_{i}}\left\{\frac{2 M}{\sqrt{h_{i}}}, \sqrt{\frac{\tau_{i}}{\varepsilon}}\right\}+h_{i}^{1 / 2}\right)\left\|\bar{\varphi}_{i, \Omega_{i}}-\varphi_{i, \Omega_{i}}\right\|_{\infty, \Omega_{i}}\left\|\varphi_{i, \Omega_{i}}^{*}-\bar{\varphi}_{i, \Omega_{i}}\right\|_{\varepsilon, \Omega_{i}},
\end{aligned}
$$

where we let $\psi_{i}^{*}=\varphi_{i, \Omega_{i}}^{*}-\bar{\varphi}_{i, \Omega_{i}}$ in Lemma A.7.

A.3.2. Error bounds. Now we are ready to get the $\varepsilon$-uniform estimate in the energy norm.

Theorem A.9. Let $\varphi_{i, \Omega_{i}}$ be the solution of (A.1) and $\varphi_{i, \Omega_{i}}^{*}$ be the solution of (A.14). Then

$$
\left\|\varphi_{i, \Omega_{i}}^{*}-\varphi_{i, \Omega_{i}}\right\|_{\varepsilon, \Omega_{i}} \leq h_{i} \gamma_{i, \varepsilon}^{*}(M) \quad \text { where } \quad \gamma_{i, \varepsilon}^{*}(M)=M^{-1}\left(\ln \left(M / h_{i}\right)\right)^{2} .
$$

Proof. Since

$$
\left\|\varphi_{i, \Omega_{i}}^{*}-\varphi_{i, \Omega_{i}}\right\|_{\varepsilon, \Omega_{i}} \leq\left\|\bar{\varphi}_{i, \Omega_{i}}-\varphi_{i, \Omega_{i}}\right\|_{\varepsilon, \Omega_{i}}+\left\|\varphi_{i, \Omega_{i}}^{*}-\bar{\varphi}_{i, \Omega_{i}}\right\|_{\varepsilon, \Omega_{i}}
$$

the result can be obtained by combining Lemmas A.4 and A.8.

The next theorem gives an $\varepsilon$-uniform estimate of $\left(\varphi_{i, \Omega_{i}}-\varphi_{i, \Omega_{i}}^{*}\right)$ in the maximum norm. 
Theorem A.10. Let $\varphi_{i, \Omega_{i}}$ be the solution of (A.1) and $\varphi_{i, \Omega_{i}}^{*}$ be the solution of (A.14). Then

$$
\left\|\varphi_{i, \Omega_{i}}^{*}-\varphi_{i, \Omega_{i}}\right\|_{\infty, \Omega_{i}} \leq h_{i} \gamma_{i, \infty}^{*}(M), \text { where } \gamma_{i, \infty}^{*}(M)=M^{3 / 2} \bar{\gamma}_{i, \infty}(M) .
$$

Proof. First, consider the fine mesh points $\left\{x_{i, k}^{*}\right\}_{k=\frac{M}{2}}^{M}$ which contains a neighborhood of the boundary layer. Using the Cauchy-Schwarz inequality with some rearrangements, we have

$$
\begin{aligned}
& \left|\left(\varphi_{i, \Omega_{i}}^{*}-\varphi_{i, \Omega_{i}}\right)\left(x_{i, k}^{*}\right)\right|=\left|\left(\varphi_{i, \Omega_{i}}^{*}-\varphi_{i, \Omega_{i}} \pm \bar{\varphi}_{i, \Omega_{i}}\right)\left(x_{i, k}^{*}\right)\right|=\left|\left(\varphi_{i, \Omega_{i}}^{*}-\bar{\varphi}_{i, \Omega_{i}}\right)\left(x_{i, k}^{*}\right)\right| \\
= & \left|\int_{x_{i, k}^{*}}^{x_{i}}\left(\varphi_{i, \Omega_{i}}^{*}-\bar{\varphi}_{i, \Omega_{i}}\right)^{\prime}(s) d s\right| \leq\left(\frac{1}{\varepsilon} \int_{x_{i, k}^{*}}^{x_{i}} d s\right)^{\frac{1}{2}}\left(\varepsilon \int_{x_{i, k}^{*}}^{x_{i}}\left|\left(\varphi_{i, \Omega_{i}}^{*}-\bar{\varphi}_{i, \Omega_{i}}\right)^{\prime}\right|^{2} d s\right)^{\frac{1}{2}} \\
\leq & \sqrt{\frac{\tau_{i}}{\varepsilon}}|| \varphi_{i, \Omega_{i}}^{*}-\left.\bar{\varphi}_{i, \Omega_{i}}\right|_{\varepsilon, \Omega_{i}} .
\end{aligned}
$$

For the coarse mesh points $\left\{x_{i, k}^{*}\right\}_{k=0}^{\frac{M}{2}}$, i.e., outside of the boundary layer, we have $h_{k}^{*} \geq \frac{h_{i}}{M}$, which implies that $1 \leq \frac{M}{h_{i}} h_{k}^{*}$ and so that,

$$
\begin{aligned}
\left|\left(\varphi_{i, \Omega_{i}}^{*}-\varphi_{i, \Omega_{i}}\right)\left(x_{i, k}^{*}\right)\right|^{2} & \leq \frac{M}{h_{i}} h_{k}^{*}\left|\left(\varphi_{i, \Omega_{i}}^{*}-\varphi_{i, \Omega_{i}}\right)\left(x_{i, k}^{*}\right)\right|^{2} \leq \frac{M}{h_{i}} \sum_{k=1}^{M-1} \bar{h}_{k}^{*}\left|\left(\varphi_{i, \Omega_{i}}^{*}-\varphi_{i, \Omega_{i}}\right)\left(x_{i, k}^{*}\right)\right|^{2} \\
& =\frac{M}{h_{i}}\left\|\varphi_{i, \Omega_{i}}^{*}-\varphi_{i, \Omega_{i}}\right\|_{L_{2}, d, \Omega_{i}}^{2} \leq \frac{2 M}{h_{i}}\left\|\bar{\varphi}_{i, \Omega_{i}}-\varphi_{i, \Omega_{i}}^{*}\right\|_{\varepsilon, \Omega_{i}}^{2} . \quad \text { (A.23) }
\end{aligned}
$$

Here we have used the following inequality,

$$
\begin{gathered}
\left\|\varphi_{i, \Omega_{i}}^{*}-\varphi_{i, \Omega_{i}}\right\|_{L_{2}, d, \Omega_{i}}^{2} \leq 2\left(\left\|\varphi_{i, \Omega_{i}}-\bar{\varphi}_{i, \Omega_{i}}\right\|_{L_{2}, d, \Omega_{i}}^{2}+\left\|\bar{\varphi}_{i, \Omega_{i}}-\varphi_{i, \Omega_{i}}^{*}\right\|_{L_{2}, d, \Omega_{i}}^{2}\right) \\
=2\left\|\bar{\varphi}_{i, \Omega_{i}}-\varphi_{i, \Omega_{i}}^{*}\right\|_{L_{2}, d, \Omega_{i}}^{2} \leq 2\left\|\bar{\varphi}_{i, \Omega_{i}}-\varphi_{i, \Omega_{i}}^{*}\right\|_{L_{2}, \Omega_{i}}^{2} \leq 2\left\|\bar{\varphi}_{i, \Omega_{i}}-\varphi_{i, \Omega_{i}}^{*}\right\|_{\varepsilon, \Omega_{i}}^{2} .
\end{gathered}
$$

Combining (A.23) and Lemma A.8, we obtain the required result. 